\title{
Collimation of high current fast electrons in dense plasmas with a tightly focused precursor intense laser pulse
}

\author{
H Xu ${ }^{1,2, \dagger}, \mathbf{X}$ H Yang ${ }^{3,4, *}, \mathbf{Z}$ M Sheng ${ }^{2,5}, \mathbf{P} \mathbf{M c K e n n a}^{5}$, Y Y $\mathbf{M a}^{3,2}$, \\ H B Zhuo ${ }^{3,2}$, Y Yin ${ }^{3}$, C Ren ${ }^{4}$, and J Zhang ${ }^{2}$ \\ ${ }^{1}$ College of Computing Science, National University of Defense Technology, Changsha \\ 410073, P. R. China \\ ${ }^{2}$ IFSA Collaborative Innovation Center, Shanghai Jiao Tong University, Shanghai \\ 200240, P. R. China \\ ${ }^{3}$ Department of Physics, National University of Defense Technology, Changsha \\ 410073, P. R. China \\ ${ }^{4}$ Department of Mechanical Engineering, University of Rochester, Rochester, New \\ York 14627, USA \\ ${ }^{5}$ Department of Physics, SUPA, University of Strathclyde, Glasgow G4 0NG, UK \\ E-mail: xuhan_email@aliyun.com \\ E-mail: xhyang@nudt.edu.cn
}

\begin{abstract}
High current fast electrons at the megaampere level provide a unique way to generate high energy density states of matter, which are related to many applications. However, the large divergence angle of the fast electrons typically over 50 degrees is a significant disadvantage for applications. The guiding effect by the self-generated azimuthal magnetic fields of the fast electron current is found to be very limited due to their cone-shaped spatial structure. In this work, we present a new understanding on the collimation conditions of fast electrons under such a magnetic field structure. It is shown that the transverse peak position of the magnetic field layer plays a crucial role to collimate the fast electrons rather than its magnitude. Based upon this, a new two-pulse collimating scheme is proposed, where a guiding precursor pulse is adopted to form proper azimuthal magnetic fields and a main pulse is for fast electron generation as usual. The present scheme can be implemented relatively easily with the precursor lasers at the $10 \mathrm{TW}$ level with a duration of two hundred femtoseconds, with which the divergence angle of fast electrons driven by the main pulse can be confined within a few degrees. Our scheme can find practical applications in high energy density science.
\end{abstract}

PACS numbers: 52.25.Fi, 52.57.Kk, 52.65.Ww

Submitted to: Nuclear Fusion 


\section{Introduction}

The ultra-short high power lasers based upon the CPA technology [1] have enabled broad applications from fundamental science to industry and medicine. In particular, such lasers push nonlinear optics to the relativistic regime [2], which can drive matters into high energy density states [3] with many applications, such as electron and ion acceleration $[4,5]$, compact x-ray $[6,7]$, neutron sources and isotope production [8-11], the fast ignition approach to inertial confinement fusion [12-14], and laboratory astrophysics [15], etc. Many of these applications mentioned above are associated with the high current relativistic electrons produced during the intense laser interaction with solid targets. Even though the efficiency of energy transfer from relativistic intense lasers to hot electrons can be well above 10\% [16], the hot electrons often are produced with large divergence angle typically over 50 degrees [17], which make it difficult to achieve collimated propagation in solid target. Collimated propagation of fast electrons is crucial for these applications. So far this has not yet been completely solved.

The penetration depth and energy deposition of fast electrons in targets strongly depend on the material properties (like electrical resistivity) and fast electron beam properties. However, the fast electrons from laser-solid interaction typically have broad angular distributions due to the beam transport in dense plasma [18-20], the laser transverse ponderomotive force [21] or the reflection of the laser light [22] in preplasmas. Generally, the divergence angle increases with the laser intensity [23]. On the other hand, the resistive magnetic fields are self-generated in the solid target, which are azimuthal and hence produce some collimating effect on the electron beam [24,25]. With this, the propagation characteristic of fast electrons can be modulated by using some specially designed targets, such as targets consisted of alternating layers of different $\mathrm{Z}$ materials $[26,27]$ and low- $\mathrm{Z}$ targets doped with high- $\mathrm{Z}$ elements [28]. It is also reported that the resistivity at low temperature (e.g., several eV), which also relies on the material microstructure, has a significant effect on the resistive magnetic field and thus the propagation of the fast electrons $[29,30]$. The collimating propagation of fast electrons can also be achieved by optimizing the parameters of laser pulses, e.g., a two-pulse injecting scheme, named guiding pulse and main pulse, was introduced theoretically [31] and later implemented in experiments [32,33]. Furthermore, multiple laser pulses with gradually increasing intensity can also enhance the collimation of fast electrons [34].

The collimation criterion of fast electrons by the self-generated magnetic field was firstly proposed by Bell and Kingham [35] and extended by Robinson et al. [31] and Curcio et al. [36], in which the spatial transverse extent $L_{\perp}$ of the magnetic field (normal to the laser propagation direction) is assumed to be equal to the focal spot size and the spatial longitudinal extent $L_{\|}$is assumed to be infinite. Therefore the criterion is intrinsically based on a one-dimensional cylindrical structure of the magnetic field. In reality, however, a cone-shaped magnetic field structure distributed at the periphery of the laser focal spot with a tilt angle is ubiquitously observed in many numerical 
simulations and experiments $[24-32,35,37-39]$. The collimation criterion with such a magnetic field structure has not been considered and understood yet. In recent years, there has been significant interest to collimate fast electrons by use of high magnetic fields [40-43]. The magnetic fields can be as high as $1 \mathrm{kT}$ when nanosecond driving lasers with energy at $\mathrm{kJ}$ level are applied $[42,44]$. This has a high demand for the driving lasers and is not easily accessible for many users.

In this paper, we investigate the conditions for fast electron collimation in the presence of a typical cone-shaped magnetic field structure driven by an ultrashort intense laser pulse, which can have a lower peak power than that used to produce fast electrons. Firstly, the magnetic field structure and its dependence on the laser parameters are studied by using a "rigid-beam" model, i.e., the fast electron current density is fixed [37]. It is found that the magnetic field structure has a specific position and a tilt angle. The transverse peak of the magnetic field layer moves to outer radii of the laser focal spot and its tilt angle approaches the initial divergence angle of fast electrons as the laser intensity increases. All these features prevent the fast electrons from being collimated by the self-generated magnetic field. To recover effective collimation, a new two-pulse collimating scheme is proposed, in which the guiding pulse has a much smaller focal spot than that of the main pulse to produce the suitable magnetic field structure. Even though the transverse peak position of the cone-shaped magnetic field generated by the guiding pulse is still larger than the focal spot radius of the guiding pulse, it is smaller than the focal spot radius of the main pulse and thus can effectively collimate the fast electrons generated by the main pulse. Finally, three-dimensional (3D) hybrid particle-in-cell (PIC)/fluid simulations are employed to validate this two-pulse scheme. It is shown that the divergence angle of the fast electron beam can be reduced from $25^{\circ}$ to $7^{\circ}$ (half-width at half-maximum: HWHM) by using a guiding pulse of $10 \mathrm{TW}$ level table-top laser system.

\section{Theoretical model for cone-shaped magnetic fields driven by intense laser}

High current fast electrons at the megaampere level can be driven by tens of terawatts intense laser pulse irradiating a solid target (see Fig. 2a for the schematic of the lasertarget interaction), which can induce megagauss magnetic fields to confine the fast electron propagation $[37,38]$. The magnetic field structure depends on the laser pulse parameters, e.g., laser intensity and pulse duration, and can be obtained by using the "rigid-beam" model. The driven current density are assumed to have a Gaussian profile (having only $z$ component $\boldsymbol{J}_{f}=J_{f} \hat{\boldsymbol{e}}_{z}$ ), varing with beam radius $r$ and propagating with the speed of light $c$, that is

$$
J_{f}(r, z, \tau)=J_{0} \frac{R_{0}}{R_{z}} \exp \left(-\frac{2 r^{2}}{R_{z}^{2}}\right) f(\tau),
$$

where $f(\tau)$ is the temporal profile, $\tau=t-z / c$ is the pulse duration (time passing through a given point). $\left.J_{0} \equiv J_{f}\right|_{r=0, z=0}$ is the current density at $z=0$ plane that is 
given by the conservation of energy flux $\beta e I_{0}=J_{0}\langle E\rangle$, where $\beta$ is absorption coefficient (usually between 0.2-0.4), $\langle E\rangle$ is the averaged energy of fast electrons determined by Wilks' scaling law [45] $\langle E\rangle /\left(m_{e} c^{2}\right)=\sqrt{1+I_{0} \lambda_{0}^{2} / 1.38 \times 10^{18}}-1, I_{0}$ is the laser peak intensity in units of $\mathrm{W} / \mathrm{cm}^{2}, \lambda_{0}$ is the wavelength in units of $\mu \mathrm{m}, R_{z}=R_{0}+z \cdot \tan (\theta)$ is the spot size of fast electron beam at $z, R_{0}$ is the spot radius at the incident plane $(z=0)$, and $\theta$ is the half-divergence of the fast electron beam.

For a high temperature plasma, e.g., $T_{e}>50 \mathrm{eV}$ for $\left(\mathrm{CH}_{2}\right)$, the Spitzer-Härm resistivity [46] $\eta\left(T_{e}\right)=\eta_{0}\left(T_{e} / T_{0}\right)^{-3 / 2}$ is a good approximation, where $T_{0}$ and $\eta_{0}$ are the initial temperature and resistivity, respectively. On the assumption of the balance between the fast electron current and the cold return current, and assuming $f(\tau)=1$ during the laser pulse period $0 \leq \tau \leq \tau_{L}$, the azimuthal component of magnetic field becomes [47]

$$
B_{\phi}=\frac{4 r}{R_{z}^{2}} \frac{C_{v} T_{0}}{J_{f}}\left[1-\frac{1+0.5 \tau^{\prime}}{\left(1+2.5 \tau^{\prime}\right)^{0.6}}\right],
$$

where $C_{v}=\frac{3}{2} \bar{Z} n_{i}$ is the electron specific heat capacity, $n_{i}$ is the ion density, $\bar{Z}$ is the averaged ionization degree of the material. Both $n_{i}$ and $\bar{Z}$ are assumed to be constants here. The normalized variable $\tau^{\prime}=\tau / \tau_{T}$ measures the heating rate of the target [37], where $\tau_{T} \equiv C_{v} T_{0} / \eta_{0} J_{f}^{2}$ is the typical heating time to temperature $T_{0}$.

The transverse peak position of the magnetic field $B_{\phi}(r, z)$ lies at $\partial B_{\phi} / \partial r=0$. By taking the derivation of Eq. (2) and some operations, the transverse peak position $r_{m}$ of the magnetic field is approximately govern by

$$
\tau^{\prime}\left(r_{m}, z\right) \simeq \frac{1}{3}
$$

Substituting Eq. (1) and the definition of $\tau^{\prime}$ into Eq. (3), then taking natural logarithm on both sides of the equation, the peak position $r_{m}$ explicitly reads

$$
\frac{r_{m}(z)}{R_{z}}=\frac{1}{2} \sqrt{\ln \left[3 \tau_{0,0}^{\prime}\left(1-\frac{z}{c t}\right)\right]+2 \ln \left(R_{0} / R_{z}\right)},
$$

where $\left.\tau_{0,0}^{\prime} \equiv \tau^{\prime}\right|_{r=0, z=0}=C_{v} T_{0} t / \eta_{0} J_{0}^{2}$. Substituting Eqs. (1) and (4) into Eq. (2), the peak magnitude of $B_{\phi}$ at plane $z=0$ reads

$$
B_{\phi, m}=0.66 \sqrt{\tau_{0,0}^{\prime} \ln \left(3 \tau_{0,0}^{\prime}\right)} \cdot C_{v} T_{0} / R_{0} J_{0}
$$

The relation of $\tau_{0,0}^{\prime}$ with laser intensity is given by [37]

$$
\tau_{0,0}^{\prime}=8.48 \times 10^{19} \frac{\eta_{0}^{5 / 3} \beta^{2} I_{0} \tau_{L}}{(\bar{Z} \ln \Lambda)^{2 / 3} n_{e} \lambda_{0}^{2}},
$$

where $\beta=0.25, \ln \Lambda$ is the Coulomb logarithm, $\eta_{0}=2.3 \mu \Omega \cdot \mathrm{m}$ and $\bar{Z} \ln \Lambda=7.7$ is used here for $\mathrm{CH}_{2}$ [48]. The normalized heat rate is about $\tau_{0,0}^{\prime} \simeq 190$ for $I_{0}=1.0 \times 10^{19} \mathrm{~W} / \mathrm{cm}^{2}$ and $\tau_{L}=1$ ps. A strong $B_{\phi, m} \propto \sqrt{I_{0} \tau_{L} \ln \left(3 I_{0} \tau_{L}\right)}$ can be achieved via the increase of the pulse intensity $I_{0}$ or pulse duration $\tau_{L}$. 

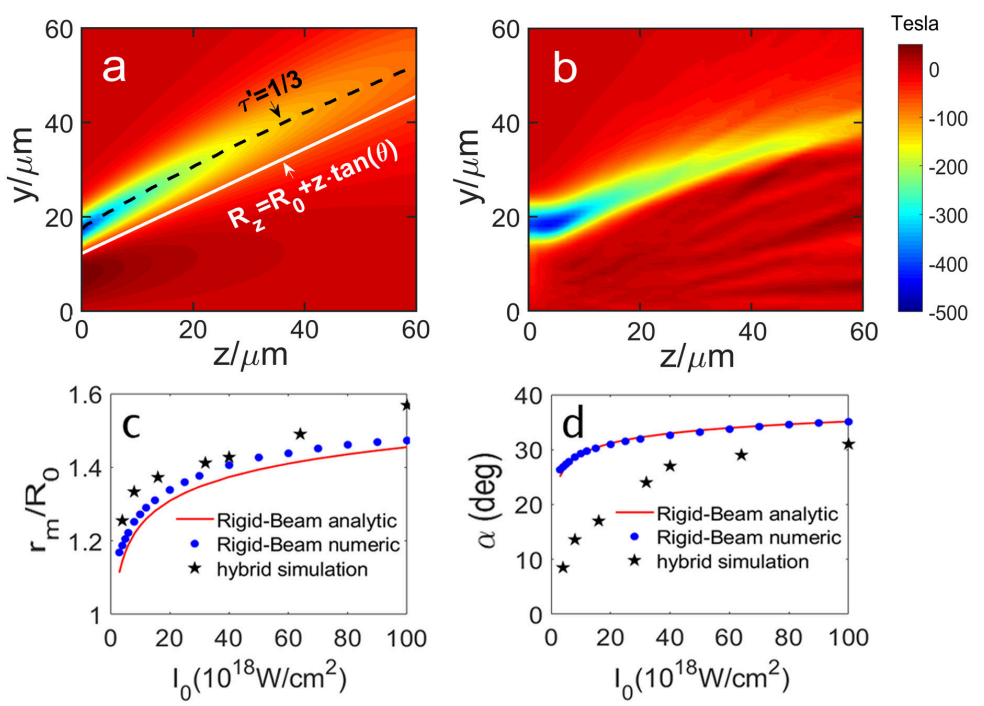

Figure 1. Distributions of the azimuthal magnetic field $B_{\phi}$ at $t=1.0 \mathrm{ps}$ obtained from a numeric solution of the "rigid-beam" model (a) and a 3D hybrid PIC/fluid simulation (b). The black dashed line and white solid line in (a) are the location of $\tau^{\prime}(r, z)=1 / 3$ and the fast electron spot radius $R_{z}$ at $z$, respectively. The transverse peak position $r_{m}$ of the magnetic field at $z=0$ plane (c) and the averaged tilt angle $\alpha$ (d) near the focal spot. The "rigid-beam" model has the identical parameters to that in the hybrid simulation described in the text.

The tilt angle $\alpha$ of the magnetic field layer can be estimated by $\tan (\alpha) \approx$ $\left[r_{m}\left(z_{1 / 2}\right)-r_{m}(0)\right] / z_{1 / 2}$, where $z_{1 / 2}$ is the longitudinal position for $B_{\phi, m} / 2$, and $r_{m}(0)$ and $r_{m}\left(z_{1 / 2}\right)$ are the transverse position of $B_{\phi, m}$ and $B_{\phi, m} / 2$, respectively. Applying Eqs. (1), (2), and (4), and since $z / c t \ll 1$ near the focal spot, the tilt angle can be approximated as

$$
\tan (\alpha) \approx \tan (\theta) \frac{\sqrt{A}}{4} \frac{1-2 x_{0}}{\sqrt{x_{0}}-x_{0}}
$$

where $x_{0}=\left[2.39-A+\sqrt{A^{2}-0.78 A+5.7}\right] / 8$ and $A \equiv \ln \left(3 \tau_{0,0}^{\prime}\right)$. For a high intensity and/or a long duration of laser pulse, e.g., $I_{0}>1 \times 10^{19} \mathrm{~W} / \mathrm{cm}^{2}$ and $\tau_{L}=1.0 \mathrm{ps}$, it shows $\tau_{0,0}^{\prime} \gg 1$ and $x_{0} \rightarrow 1 / 4$, which leads to $r_{m}(0)=\frac{\sqrt{A}}{2} R_{0}$ and $\tan (\alpha)=\frac{\sqrt{A}}{2} \tan (\theta)$, i.e., we generally have

$$
r_{m}(0)>R_{0}, \quad \alpha>\theta .
$$

This means that the self-generated magnetic field layer is located outside of the laser focal spot. Due to the transverse Gaussian profile of the main laser pulse, only $4.5 \%$ of fast electrons are located out of the focal spot radius $\left(r>R_{0}\right)$ and $0.6 \%$ of fast electrons are located out of the magnetic field layer $\left(r>1.36 R_{0}\right.$, see Fig. 1c). Thus, most of the fast electrons have no chance to enter into the magnetic field area and to be collimated by the field, although the magnitude of the peak magnetic field is still strong enough (up to 500T) to collimate the fast electrons according to the estimation of Robinson et al. [31]. 
Figure 1 shows the structure of the self-generated magnetic field from "rigid-beam" model (Fig. 1a) and a 3D hybrid simulation (Fig. 1b). In both cases, we set the peak intensity $I_{0}=4 \times 10^{19} \mathrm{~W} / \mathrm{cm}^{2}$, spot radius $R_{0}=12.75 \mu \mathrm{m}$ (corresponding to full-width at half-maximum (FWHM) of $15 \mu \mathrm{m})$, pulse duration $1.0 \mathrm{ps}$, and the half-divergence angle $30^{\circ}$. The target is polyethylene $\left(\mathrm{CH}_{2}\right)$ with a uniform density of $1.0 \mathrm{~g} / \mathrm{cm}^{3}$. An initial electron temperature of $48.3 \mathrm{eV}$ is set to keep the initial resistivity $2.3 \mu \Omega \cdot \mathrm{m}$ close to that used in reference [48]. Figure $1 \mathrm{a}$ and $1 \mathrm{~b}$ show a similar magnetic field structure, both appearing within a narrow band at the periphery of the focal spot. The tilt angle $\left(32.6^{\circ}\right)$ in Fig. 1a is larger than that of the hybrid simulation in Fig. $1 \mathrm{~b}\left(27^{\circ}\right)$. The black dashed line in Fig. 1a is the location of the peak position $r_{m}$ of $B_{\phi}$ defined by Eq. (3), which shows a good agreement with the numerical result.

The dependence of $r_{m}$ and the tilt angle $\alpha$ on laser intensities are shown in Figs. $1 \mathrm{c}$ and $1 \mathrm{~d}$. It is seen that both the $r_{m}$ and $\alpha$ increase with laser intensity, and the analytical results (Eqs. (4) and (7)) are in good agreement with the numerical results of the "rigid-beam" model. Note that the tilt angle $\alpha$ from the hybrid simulations is much

smaller than that from the "rigid beam" model. This is due to the feedback missing of the collimation effect of the fast electrons in the "rigid beam" model, especially at lower laser intensities.

\section{Conditions for fast electron collimation by the cone-shaped magnetic fields}

To investigate the confinement of fast electrons by the self-generated magnetic field with a cone-shaped distribution, a simplified structure of the self-generated magnetic field is adopted as shown in Figs. 2. The magnetic flux density $B_{0}$ is uniformly distributed with spatial length of $L$, thickness of $d$, and tile angle of $\alpha$. It covers the main features of the magnetic field obtained from the "rigid-beam" model (Fig. 1a) and hybrid simulations (Fig. 1b). We consider test fast electrons, which are injected from the left boundary with an initial angle of $\theta$ and a transverse offset $R$ to the the magnetic field layer. The trajectory of the fast electron (red lines with arrows) is a part of circle (grey dashed-line in Fig. 2a) in a uniformly perpendicular magnetic field, where $r_{0}=\gamma_{0} m_{e} v_{0} / e B_{\phi}$ is the Larmor radius, and $v_{0}, e, m_{e}, \gamma_{0}$ are the fast electron velocity, charge, mass, and Lorentz factor, respectively.

Note that there are only four cases that the fast electron can be confined by the magnetic field. For the first two cases, the injecting angles of the fast electrons are greater than the tilt angle of the magnetic field (i.e, $\alpha<\theta$ ) but with a different transverse offsets $R$ are shown in Figs. 2a and 2c. For the other two cases, the fast electrons with $\alpha>\theta$ are shown in Figs. 2b and 2d. By considering the geometrical relationship between the fast electron trajectory and magnetic field structure, one can obtain the 

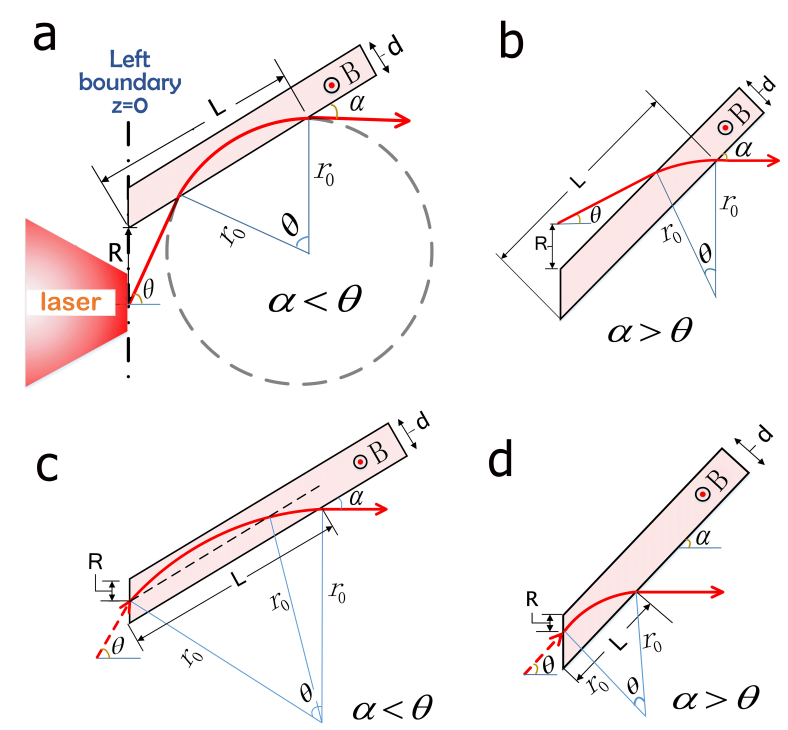

Figure 2. (Color online) Sketch of fast electrons deflected/collimated by an azimuthal magnetic field $B_{\phi}$. The magnetic field is azimuthal and points outward, with a tilt angle $\alpha$, spatial length of $L$, and thickness of $d$. The laser incidents from the left boundary (thick dashed-dot line in (a)) and produces a sampled fast electron, which has an initial divergence angle of $\theta$ and an initial transverse offset $R$ to the lower/upper edge of the magnetic layer. The trajectory of fast electron is marked by a red line with an arrow and $r_{0}$ is the Larmor radius of the fast electron.

fast electron collimating conditions for the above four cases,

$$
\begin{aligned}
& \text { Case a : } \begin{cases}d> & d_{1}, \\
L> & 2 r_{0} \sin (\theta / 2)+L_{2},\end{cases} \\
& \text { Case b : } \begin{cases}d> & d_{2}, \\
L> & L_{1}+L_{2},\end{cases} \\
& \text { Case c : } \begin{cases}d> & d_{2}+R \cos (\alpha), \\
L> & L_{1}, \\
R> & d_{1} / \cos (\alpha),\end{cases} \\
& \text { Case d : } \begin{cases}d> & d_{2}+R \cos (\alpha), \\
L> & L_{1} .\end{cases}
\end{aligned}
$$

where $d_{1}=r_{0}[1-\cos (\theta / 2)], d_{2}=r_{0}[\cos (\alpha-\theta)-\cos (\alpha)], L_{1}=r_{0} \sin (\theta) / \cos (\alpha)$, and $L_{2}=R \cos (\theta) / \sin (|\theta-\alpha|)$. These conditions define the required magnetic field sizes to achieve electron collimation. One notes that Eq. (8a) reduces to the result of Robinson et al. when $\alpha=0$ and $R=0$ [31]. When the tilt angle of magnetic field approaches the electron divergence, i.e., $\alpha \rightarrow \theta$, then $L_{2} \rightarrow \infty$, thus $L$ in case (a) and case (b) become very large, which leads to that only cases (c) and (d) are effective to confine the fast electrons. The fast electrons would be collimated by the magnetic field if only they can 
satisfy one of Eqs. (8a)-(8d). Once the initial relative position $r$ (in Fig. 2) of the fast electrons and the structure of magnetic field are given, the fraction of collimated fast electrons can be estimated.

For the more realistic case, the collimation effect of fast electrons by the coneshaped magnetic field needs to be evaluated by numerical simulation. We follow the trajectories of a total of $10^{6}$ fast electrons, sampled with different $r$ according to the transverse Gaussian distribution function $\exp \left(-2 r^{2} / R_{0}^{2}\right)$, which are bent in the selfgenerated magnetic field obtained numerically from the above "rigid-beam" model. The results are shown as the "circle-black-dashed-line" and "circle-black-solid-line" in Fig. 3 for two different pulse durations. It can be seen that the fraction of collimated fast electrons decreases rapidly with the increase of the laser intensity. This can be attributed to two reasons: Firstly, the energy of the fast electrons increases with laser intensity according to Wilk's scaling law, indicating that a stronger magnetic field to be required to collimate the fast electrons; Secondly, both the transverse position $\left(r_{m} / R_{z}\right.$ in Eq. (4) and Fig. 1c) and the tilt angle ( $\alpha$ in Eq. (7) and Fig. 1d) of peak magnetic field increase, leading to that less fast electrons can be deflected. Actually, fast electrons are hardly collimated by the self-generated magnetic field alone as laser intensity $I_{0}>3 \times 10^{19} \mathrm{~W} / \mathrm{cm}^{2}$ (i.e., less than $1 \%$ ). All these suggest that fast electrons cannot be collimated by the self-generated magnetic fields themselves.

In order to confine the fast electrons effectively for a high laser intensity, we propose a two-pulse scheme by use of a guiding pulse and a main pulse, in which the guiding laser pulse with a much smaller focal spot than the main pulse is first incident to generate a cone-shaped magnetic field structure and then the main pulse is injected. The pregenerated magnetic field structure has a size larger than the focal spot of guiding pulse but still smaller than the spot of the main pulse, which enable to collimate the fast electrons generated by the main pulse. With this scheme, the fast electrons with incident angle $\theta<\alpha$ can be collimated under the cases shown in Figs. 2b and 2d, while the fast electrons with incident angle $\theta>\alpha$ will be collimated under the cases shown in Figs. $2 \mathrm{a}$ and 2c. To demonstrate the effectiveness of this scheme, we carry out numerical simulations based upon the "rigid-beam" model as shown above. Similarly, a total of $10^{6}$ test fast electrons are checked for this scheme. Figure 3 shows the ratio of collimated fast electrons against the total number of injected electrons under different focal spot sizes and pulse durations of the guiding laser pulse. One can seen that, by applying a guiding pulse with a smaller focal spot radii $\left(R_{0} / 2\right.$ and $R_{0} / 3$, respectively), the fraction of collimated fast electrons increase drastically for a much stronger laser intensity. In addition, the fraction of collimated fast electrons can be enhanced by increasing the duration of the guiding pulses. Furthermore, such collimation becomes more efficient as the spot radius decreases from $R_{0} / 2$ to $R_{0} / 3$. It is shown that the collimated electron number is increased by $\sim 16$ times for the case with a laser intensity of $3 \times 10^{19} \mathrm{~W} / \mathrm{cm}^{2}$ and a guiding pulse with a spot radius of $R_{0} / 3$ and duration of $1 \mathrm{ps}$ compared to that of without the guiding pulse. 


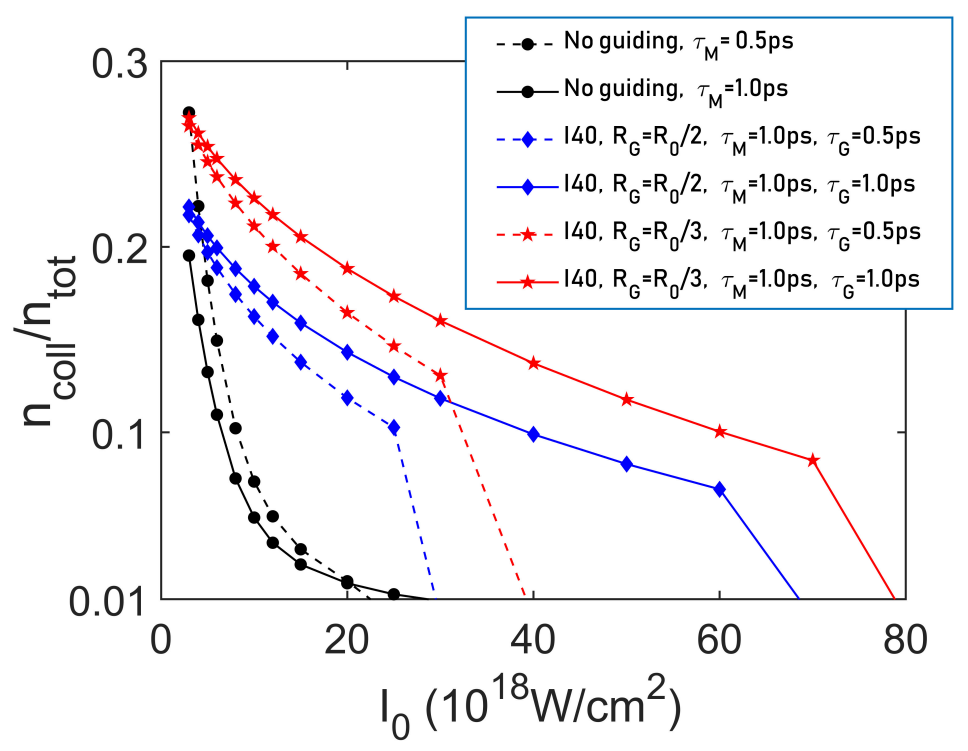

Figure 3. The fraction of collimated fast electrons for cases with/without precursor guiding pulses. $n_{\text {coll }}$ and $n_{\text {tot }}$ are the numbers of collimated and total injected fast electrons, respectively. $\tau_{G}$ and $\tau_{M}$ are the pulse durations of the guiding pulse and main pulse, respectively. The "circle-black-dashed-line" and "circle-black-solid-line" are the results without the guiding pulse at $\mathrm{t}=0.5 \mathrm{ps}$ and $1.0 \mathrm{ps}$, respectively. The "diamond-blue-line" and "pentagram-red-line" correspond to the cases with the guiding pulses with spot radii of $R_{0} / 2$ and $R_{0} / 3$, respectively, where $R_{0}=12.75 \mu \mathrm{m}$ is the spot radius of the main pulse. Here I40 means the intensity of guiding pulse is $40 \times 10^{18}$ $\mathrm{W} / \mathrm{cm}^{2}$.

\section{3D HYBRID SIMULATIONS}

In order to validate the two-pulse scheme further, some 3D hybrid PIC/fluid simulations are carried out by using the code HEETS [28], which treats the fast electrons as a fully dynamical method including collisions (i.e., explicit PIC), while the background electron-ion plasmas are described by a reduced two-fluid model. The simulation box is $200 \times 200 \times 200 \mu \mathrm{m}^{3}$ with grid of $\Delta x=\Delta y=\Delta z=1 \mu \mathrm{m}$. Both the guiding pulse and the main pulse are modeled by setting identical constant intensity $I_{0}=4 \times 10^{19} \mathrm{~W} / \mathrm{cm}^{2}$, and energy efficiency from laser-to-fast electrons of $25 \%$. The main pulse having a duration of $2 \mathrm{ps}$ and a transverse Gaussian radial profile $\sim \exp \left(-2 r^{2} / R_{0}^{2}\right)$ with $R_{0}=12.75 \mu \mathrm{m}$, following the guiding pulse of a duration of $\tau_{\text {guid }}=0.2 \mathrm{ps}$, Gaussian radial profile of $R_{\text {guid }}=R_{0} / 2$ or $R_{0} / 3$, and a time delay of $0.2 \mathrm{ps}$ for the major simulation. The angular distribution of fast electrons is specified to be $g(\theta)=\cos ^{M}(\delta \theta)$, where $M=6, \delta=\cos ^{-1}\left(2^{-1 / M}\right) / \theta_{0}, \theta_{0}=\pi / 6$ is HWHM divergence angle [23] for both the guiding and main pulse. The energy distribution of fast electrons are assumed to be $h(E)=\exp (-E /\langle E\rangle) /\langle E\rangle$, where the averaged energy $\langle E\rangle$ is given by Wilks' scaling law [45]. The target is polystyrene with a uniform density of $1.0 \mathrm{~g} / \mathrm{cm}^{-3}$ and an initial temperature $1 \mathrm{eV}$. The target resistivity is $\eta=\left(1 / \eta_{\max }+1 / \eta_{S P}\right)^{-1}[48]$, where 
$\eta_{\max }=2.3 \mu \Omega \cdot \mathrm{m}$ is the maximum of the resistivity, $\eta_{S P}=7.7 \times 10^{-4} T_{e}^{-3 / 2}$ is the Spitzer-Härm resistivity [46]. The average ionization degree of the target is determined by the Thomas-Fermi model [49].

Figure 4 shows the isosurface of fast electron density (sampled at $4 \times 10^{25} \mathrm{~m}^{-3}$ ), the corresponding density distribution at the target rear surface, and self-generated magnetic fields $B_{x}$ and $B_{y}$ components for the cases with/without a guiding pulse at $t=1.6$ ps. Note $t=0$ is corresponding to the time at which the leading edge of the main pulse enters the left boundary of the simulation box. For the case without a guiding pulse (see Fig. 4a), it can be seen that the fast electrons are divergent in the target with an average divergence angle about $25^{\circ}$, which is smaller than the initial angle $30^{\circ}$ due to the weak confinement effect of the self-generated magnetic field. By employing a precursor guiding pulse, the divergence angle of fast electrons is reduced significantly, e.g., $15^{\circ}$ for a guiding pulse with the spot radius of $R_{0} / 2$ (see Fig. $4 \mathrm{~b}$ ) and only $7^{\circ}$ for the case of $R_{0} / 3$ (see Fig. 4c). One can see that, in Fig.4c, a small handlebar-like magnetic field structure (peak magnitude about $400 \mathrm{~T}$ ) appears around the focus center. Though the magnitude of this field is weaker than that due to the main pulse (about $580 \mathrm{~T}$ ), it plays an important role to confine the fast electrons due to the fact that it is located in the region of fast electron propagation. When the spot radius of the guiding pulse becomes larger, this handlebar-like magnetic field moves outward and merges with the magnetic field of the main pulse, as shown in Fig. 4b, so the confinement becomes weaker.

To investigate the influence of the parameters of the guiding pulse on the fast electron collimation, more simulations are carried out. The fast electron spot radius $R_{\text {out }}$ at the rear surface of the target and averaged target temperature $T_{\text {avg }}$ within $R_{\text {out }}$ are recorded for different spot radii (Fig. 5a), different pulse durations (Fig. 5b) of the guiding pulses, and different time-delay between the guiding pulse and main pulse (Fig. $5 \mathrm{c})$. The averaged temperature of the target is relevant to the fast electron energy flux penetrating through the rear surface, which can directly show the collimation effect. It is shown that the guiding pulse has a optimum spot radius around $5 \mu \mathrm{m}$ (FWHM). Further decreasing the spot radius will lead to the increase of number of fast electrons missing the confinement of the magnetic field. In the "rigid-beam" model, the peak magnetic field is dependent on the product of pulse duration $\tau$ and intensity $I_{0}$, i.e., $B_{\phi, m} \propto \sqrt{I_{0} \tau \ln \left(3 I_{0} \tau\right)}$ (see Eqs. (5)), which indicates that a longer pulse duration will induce a stronger magnetic field and so a better confinement, just as shown in Fig. 5b. One can note that, in Fig. 5b, a pulse duration of $200 \mathrm{fs}$ (energy 2.27 Joule with peak power of $11.35 \mathrm{TW}$ ) drastically reduces the fast electron divergence. In that case, the fast electron spot radius at the rear surface of the target is about $35 \mu \mathrm{m}$ comparing to $105 \mu \mathrm{m}$ for the case without a guiding pulse. Fig. 5c shows that the influence of time-delay between the guiding pulse and main pulse (at least within $2 \mathrm{ps}$ ) is very weak. This can be attributed the long lifetime of magnetic field structure. The characteristic diffusion length of the magnetic field is given by $L_{d}=\left(\eta \tau_{d} / \mu_{0}\right)^{1 / 2}[50,51]$, where $\eta$ is the resistivity of target, $\mu_{0}$ is the permeability of free space, $\tau_{d}$ is the time delay between the 


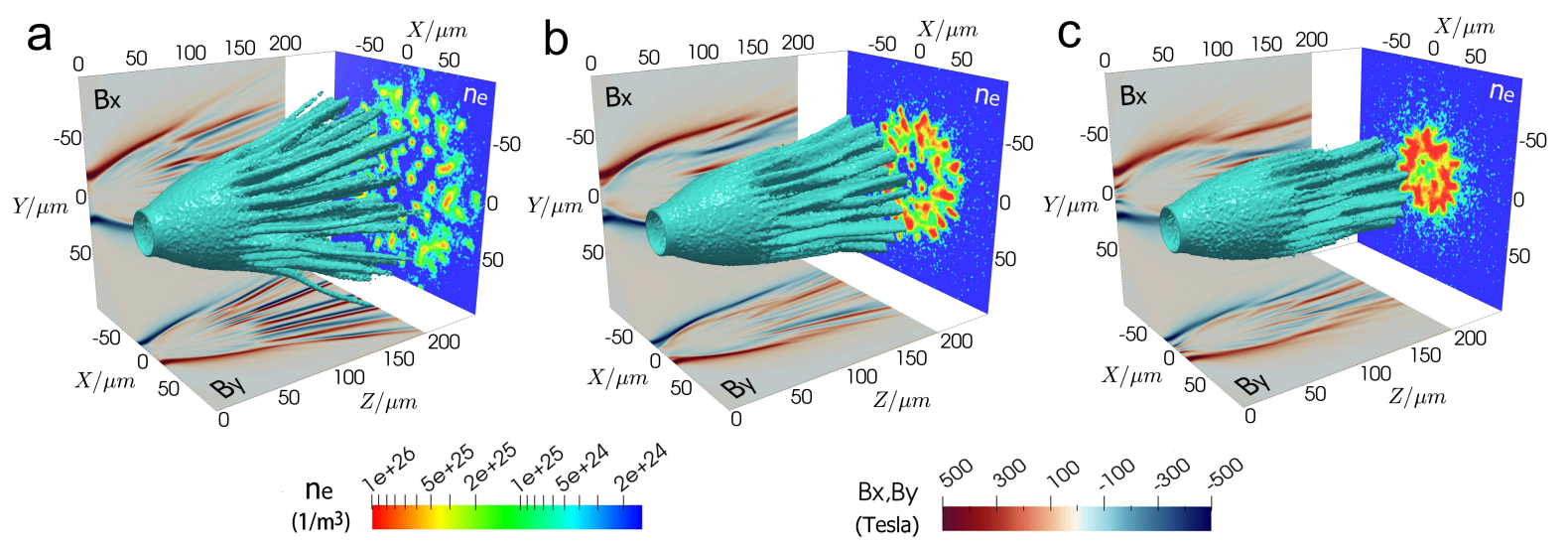

Figure 4. Isosurfaces (sampled at $4 \times 10^{24} \mathrm{~m}^{-3}$ ) and $\log _{10}$ of fast electron number density on the rear surface $z=200 \mu m$ at $t=1.6 \mathrm{ps}$ from $3 \mathrm{D}$ hybrid simulations for the cases without guiding pulse (a), guiding pulses with spot radii of $R_{0} / 2$ (b) and $R_{0} / 3$ (c) at $t=1.6 \mathrm{ps}$, respectively. Distributions of the self-generated magnetic field $B_{x}$ at $x=0$ plane and $B_{y}$ at $y=0$ plane are also presented. The density and magnetic field are in units of $\mathrm{m}^{-3}$ and tesla, respectively. $t=0$ corresponds to the leading edge of the main pulse crosses the left boundary of the simulation box. Other parameters are as follow: both the intensities of main and guiding pulses are $I_{0}=4 \times 10^{19} \mathrm{~W} / \mathrm{cm}^{2}$, the duration of main and guiding pulses are $2 \mathrm{ps}$ and $0.2 \mathrm{ps}$, respectively, the focal spot radii are $R_{0}=12.75 \mu \mathrm{m}\left(R_{0, F W H M}=15 \mu \mathrm{m}\right)$ for the main pulse, and $R_{0} / 2(\mathrm{~b})$ and $R_{0} / 3$ (c) for the guiding pulses, the delay time between guiding and main pulses is 0.2 ps.

two pulses. For materials that have been heated and ionized, with maximum resistivity around $10^{-6} \Omega \cdot \mathrm{m}$, one can find that $L_{d}$ is very small for the picoseconds time delay, e.g., $L_{d} \sim 0.4 \mu \mathrm{m}$ and $\sim 1.26 \mu \mathrm{m}$, respectively, for $\tau_{d}=0.2 \mathrm{ps}$ and $2 \mathrm{ps}$. That is, after the magnetic field is generated by the guiding pulse, the magnitude and structure of magnetic field will not have obvious variance in such short time delay. It indicates that our scheme should be robust enough to be implemented in future experiments. Such two pulses can be originated from the same oscillator and be temporally synchronized using interferometry techniques. One can use two different off-axis parabolic mirrors, one for each laser pulse, to vary the focal spot size of the pulses.

\section{CONCLUSION}

In summary, we have investigated the magnetic field structure produced during the fast electron transport in dense plasma, where the fast electrons are generated by relativistic laser interaction with solid targets. The magnetic field structure typically has a coneshape with an open angle, which increases with the driving laser intensity. It is also located outside the focal spot of the driving laser pulse. It is shown that fast electrons generated by high laser intensity are hardly confined by this self-generated magnetic field (e.g., less than $1 \%$ of the total fast electrons can be collimated as $I_{0}>3 \times 10^{19} \mathrm{~W} / \mathrm{cm}^{2}$ ) due to such magnetic field structure. The criteria for collimating fast electron beam 

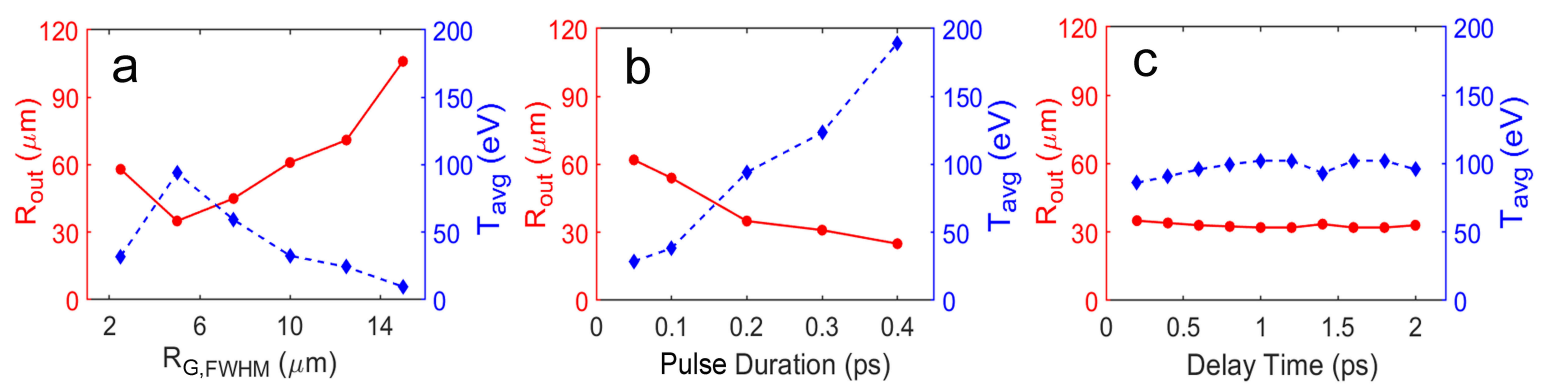

Figure 5. The spot radius of fast electrons $R_{\text {out }}$ (red $y$-axis) and the averaged electron temperature $T_{\text {avg }}$ within $R_{\text {out }}$ (blue $y$-axis) at the rear surface, for (a) different focal spot radii of the guiding pulse with duration $\tau_{G}=0.2 \mathrm{ps}$, (b) different guiding pulse duration with focal spot radius $R_{G}=R_{0} / 3$, and (c) different delay time between the guiding and main pulses with $\tau_{G}=0.2 \mathrm{ps}, R_{G}=R_{0} / 3$. Other parameters are same with that in Fig. 4.

transport in dense plasmas in the presence of a cone-shaped magnetic field structure are presented. Based upon these, a two-pulse collimating scheme is proposed, in which a precursor guiding pulse with a focal spot smaller than the main pulse is applied to generate a favorable magnetic field layer. The validity and robustness of the twopulse collimating scheme are verified by $3 \mathrm{D}$ hybrid simulations. It is shown that such a magnetic field structure driven by a $10 \mathrm{TW}$ laser can already produce significant collimating effect, where the divergence angle of fast electron beam can be decreased from $25^{\circ}$ to $7^{\circ}$ by employing a guiding pulse with a spot radius of $R_{0} / 3$. The time delay between the guiding laser pulse and the main laser pulse is relatively flexible (at least within 2 ps considered here). Thus, even a relatively low-cost compact tabletop femtosecond laser system may be helpful to collimate the fast electrons. This approach can be relatively easily adopted in experiments and thus can find many interesting applications when collimated fast electron transport is required.

\section{Acknowledgments}

This work was supported by the NNSFC (Grant Nos. 11775305, 11721091, 11774430, 11665012, and 11475260), Science Challenge Project(Grant Nos. TZ2018001 and TZ2018005), and Open Fund of the State Key Laboratory of High Field Laser Physics (SIOM). X.H.Y. also acknowledges the support from the China Scholarship Council.

\section{References}

[1] Strickland D and Mourou G 1985 Optics comm. 55, 447

[2] Mourou G A et al 2006 Rev. Mod. Phys. 78, 309

[3] Davidson R C et al 2004 Frontiers for Discovery in High Energy Density Physics

[4] Macchi A et al 2013 Rev. Mod. Phys. 85, 751

[5] Robson L et al 2007 Nature Phys. 3, 58

[6] Park H S et al 2008 Phys. Plasma. 15,072705 
[7] Jin Z et al 2011 Phys. Rev. Lett. 107, 265003

[8] Ditmire T et al 1999 Nature 398, 489

[9] Zhuo H B et al 2009 Phys. Rev. E 79, 015401(R)

[10] Xu H et al 2012 Appl. Phys. Lett. 100, 144101

[11] Ledingham K W D et al 2003 Science 300, 1107

[12] Tabak M et al 1994 Phys. Plasmas 1, 1626

[13] Horioka K, 2018 Matter and Radiation at Extremes,3, 12

[14] Campbell E M et al 2017 Matter and Radiation at Extremes,2, 37

[15] Chen H et al 2010 Phys. Rev. Lett. 105, 015003

[16] Levy M C et al 2014 Nature Comm. 5, 4149

[17] Strozzi D J et al 2012 Phys. Plasmas 19, 072711

[18] Adam J C et al 2006 Phys. Rev. Letts. 97, 205006

[19] Yang X H et al 2016 Phys. Plasmas 23, 103110

[20] Lang Y et al 2018 Plasma Phys. Control. Fusion 60, 075002

[21] Ovchinnikov V M et al 2013 Phys. Rev. Letts. 110, 065007

[22] Pérez F et al 2013 Phys. Rev. Lett. 111, 245001

[23] Green J S et al 2008 Phys. Rev. Lett. 100, 015003

[24] Davies J R et al 1997 Phys. Rev. E 56, 7193

[25] Vaisseau X et al 2017 Phys. Rev. Lett. 118, 205001

[26] Robinson A P L and Sherlock M 2007 Phys. Plasmas 14, 083105

[27] Robinson A P L et al 2012 Phys. Rev. Lett. 108, 125004

[28] Xu H et al 2019 Plasma Phys. Control. Fusion 61, 025010

[29] McKenna P et al 2011 Phys. Rev. Lett. 106, 185004

[30] MacLellan D A et al 2013 Phys. Rev. Lett. 111, 095001

[31] Robinson A P L et al 2008 Phys. Rev. Lett. 100, 025002

[32] Scott R H H et al 2012 Phys. Rev. Lett. 109, 015001

[33] Malko S et al 2019, preprint arXiv:1904.04893

[34] Volpe L et al 2014, Phys. Rev. E 90, 063108

[35] Bell A R and Kingham R J 2003 Phys. Rev. Lett. 91, 035004

[36] Curcio A and Volpe L, 2019 Plasma Phys. Control. Fusion, 61, 055013

[37] Davies J R et al 2006 Plasma Phys. Control. Fusion 48, 1181

[38] Yang X H et al 2012 Phys. Plasmas 19, 062702

[39] Romagnani L et al 2019 Phys. Rev. Lett. 122, 025001

[40] Zhuo H B et al 2014 Phys. Rev. Lett. 112, 215003

[41] Wang W M et al 2015 Phys. Rev. Lett. 114, 015001

[42] Bailly-Grandvaux M et al 2018 Nature Comm. 9, 102

[43] Yang X H et al 2018 Phys. Plasmas 25, 063104

[44] Fujioka F et al 2013 Sci. Rep. 3, 1170

[45] Wilks S C et al 1992 Phys. Rev. Lett. 69, 1383

[46] Spitzer L and Härm R 1953 Phys. Rev. 89, 977

[47] Debayle A et al 2010 Phys. Rev. E 82, 036405

[48] Davies J R et al 1999 Phys. Rev. E 59, 6032

[49] Atzeni S and Meyer-ter-Vehn J 2009 The Physics of Inertial Fusion: Beam Plasma Interaction, Hydrodynamics, Hot Dense Matter (Oxford University Press, USA)

[50] Bellan P M 2006 Fundamentals of Plasma Physics (New York: Cambridge Univ. Press)

[51] Yang X H et al 2015 Plasma Phys. Control. Fusion 57, 025011 